\title{
Impact of Predicting Real-Time Clock Corrections during their Outages on Precise Point Positioning
}

\author{
A. El-Mowafy, Dept of Spatial Sciences, Curtin University, Australia \\ PO Box U 1987, Perth WA 6845, Australia, a.el-mowafy@curtin.edu.au, Tel: +61892663403.
}

\begin{abstract}
Real-time Precise Point Positioning (RT-PPP) is a popular positioning method for natural hazard warning systems (NHWS) such as for monitoring tsunami and earthquakes. PPP relays on the use of precise orbits and clock corrections. Hence, the positioning accuracy of RT-PPP will significantly deteriorate when experiencing a discontinuity in receiving these corrections, for instance due to a temporary modem failure. The best available approach in this case would be to use the International Global Navigation Satellite Systems Service (IGS) ultra-rapid (IGU) orbits and clocks that would result in a low positioning accuracy. In this paper, we present a simplified approach to a method that we recently proposed to bridge outages in the corrections and improve the RT-PPP accuracy compared with the use of IGU. In this method, the most recent IGU orbits are used as they are compatible with the RT orbits; however, the clock corrections are predicted as a time series using a linear model with four sinusoidal terms. The prediction errors resulting from this method, age of the model and its validity period are discussed. The impact of using the proposed approach is evaluated at a number of sites of known positions by comparing its performance to using IGU orbits and clocks. Moreover, the impact of using predicted corrections is assessed in different scenarios. The experimental results proved validity of the presented approach where positioning mean RMSE of less than $20 \mathrm{~cm}$ was maintained during the outage period.
\end{abstract}

Keywords: Precise point positioning, outages, prediction, real-time positioning, GNSS

\section{Introduction}

Positioning using Global Navigation Satellite Systems (GNSS) provides an effective tool for early detection and characterising of natural hazardous events such as earthquakes, tsunamis, landslides and volcanic eruption (Blewitt et al., 2009; Rizos, 2015; Bawden et al. 2016). Such systems should be able to provide a warning as early as 5 to $10 \mathrm{~min}$ after an earthquake (Hoechner et al., 2013). Some examples of these systems are the Jet Propulsion Laboratory's GPS Real Time Earthquake and Tsunami Alert project (GREAT); the Real-Time Earthquake Analysis for Disaster mitigation network (READI); NASA-NOAA GPS-Aided Tsunami Early Detection (GATED) system; Earthquake Early Warning and Tsunami Warning of Japan, and the German-Indonesian Tsunami Early Warning System (GITEWS). These systems use a dense network of GNSS receivers at Continuous Operating Reference stations (CORS) and sea buoys, set up at strategic locations for monitoring land displacement and sea level fluctuations. The use of RT-PPP on board of ships at anchor was also proposed for disaster prevention (Saito and Kubo, 2016).

El-Mowafy, A. (2017). Impact of Predicting Real-Time Clock Corrections during their Outages on Precise Point Positioning. Survey Review, DOI 10.1080/00396265.2017.1405155. 
The key to the GNSS-dependent natural hazard warning systems is real-time precise positioning. To this end, RT-PPP is becoming a popular approach. To enable RT-PPP, the IGS launched an open access Real Time Service (RTS) in April 2013. In addition, the Japanese Aerospace Exploration Agency (JAXA) has developed a Multi-GNSS orbit and clock estimator service intended for RT-PPP, known as Multi-GNSS Advanced Demonstration tool for Orbit and Clock Analysis (MADOCA) (https://ssl.tksc.jaxa.jp/madoca/public/public_index_en.html). Moreover, a number of private commercial providers offer similar products such as Fugro G2 service (https://www.fugro.com/our-services/marine-asset-integrity/satellite-positioning/starfix), Trimble RTX service (Leandro et al. 2011), and TERRASTAR (http://www.terrastar.net/).

Since RT-PPP relays on online streaming of orbit and clock corrections, a major concern for using this method is in case when an interruption in receiving the corrections takes place, for instance due to a temporary user modem failure. In this case, a decline in PPP accuracy may result. To enable RT-PPP during such an outage, in our earlier work we proposed to predict real-time clock corrections as time series using a quadratic model with sinusoidal terms (ElMowafy et al., 2017). Yang et al. (2017) presented a similar approach. In this contribution, a simpler form of our earlier model is presented that is capable of providing a comparable performance. In addition, some critical factors in the prediction process that was not addressed before is discussed, such as age of data and model validity period. In addition, the impact of using the predicted corrections at different scenarios is demonstrated.

The next section discusses different IGS products that can be applied for PPP and their positioning accuracy referenced to a benchmark scheme when using the IGS final precise orbits and clock corrections. The impact of an outage in real-time products is demonstrated. The implementation model of the openly available IGS-RTS orbit and clock corrections is presented. Next, the proposed approach is summarised, where the clock prediction model is presented and some critical aspects in its implementation are discussed. Subsequently, the accuracy of applying the clock prediction model is assessed. Finally, test results are presented to evaluate performance of the proposed method.

\section{PPP Using IGS real-time products}

In PPP, four IGS products can be used, namely: the IGS final and rapid orbits and clocks, the IGS real-time product (IGS RTS), and the IGS Ultra-rapid product (IGU). The last two can be applied in real time. To demonstrate the impact of using each of these products on PPPP positioning accuracy, one day of GPS data (02/01/2017) at the IGS station TWFT was processed using a traditional PPP float ambiguity method (Zumberge et al., 1997). These ephemeris and clock products in addition to the broadcast ephemeris were used in separate runs using the same set of observations. The rapid products are close to the final products; therefore, it is sufficient to show results of the latter. The positioning accuracy is expressed in terms of the difference between the PPP estimated coordinates at each epoch and the known coordinates of the station. Figure 1 shows a time series of the positioning accuracy using the four products, namely: IGS final, IGS RTS, IGU and broadcast for the Easting, Northing and $\mathrm{Up}(\mathrm{E}, \mathrm{N}, \mathrm{U})$ coordinates given in a local level frame. Table 1 shows the corresponding statistics in terms of the mean of the absolute values, and the RMSE (representing accuracy as the results were referenced to the known point coordinates) for the full day of data. The main objectives here are twofold. Firstly, to show the performance when using the IGS RTS 
compared with the IGS final products, where the latter is the best product that one can use for PPP processing. Secondly, to show the impact of experiencing an outage in the IGS RTS in real-time as users switch to IGU (Haug et al., 2014), and if IGU is not accessible where they rely on the broadcast orbits and clocks.

Table 1 Average values of the position accuracy (m) using various products

\begin{tabular}{c|ccc|ccc|ccc|ccc}
\hline Product & \multicolumn{3}{|c|}{ IGS final } & \multicolumn{3}{|c|}{ IGS RTS } & \multicolumn{3}{c}{ IGU } & \multicolumn{3}{c}{ Broadcast } \\
\hline component & $\mathrm{E}$ & $\mathrm{N}$ & $\mathrm{U}$ & $\mathrm{E}$ & $\mathrm{N}$ & $\mathrm{U}$ & $\mathrm{E}$ & $\mathrm{N}$ & $\mathrm{U}$ & $\mathrm{E}$ & $\mathrm{N}$ & $\mathrm{U}$ \\
\hline $\begin{array}{c}\text { Mean } \\
\text { (absolute) }\end{array}$ & 0.058 & 0.049 & 0.124 & 0.067 & 0.039 & 0.125 & 0.253 & 0.154 & 0.316 & 0.318 & 0.385 & 0.577 \\
\hline RMSE & 0.076 & 0.062 & 0.151 & 0.080 & 0.053 & 0.156 & 0.303 & 0.219 & 0.317 & 0.383 & 0.419 & 0.653 \\
\hline
\end{tabular}
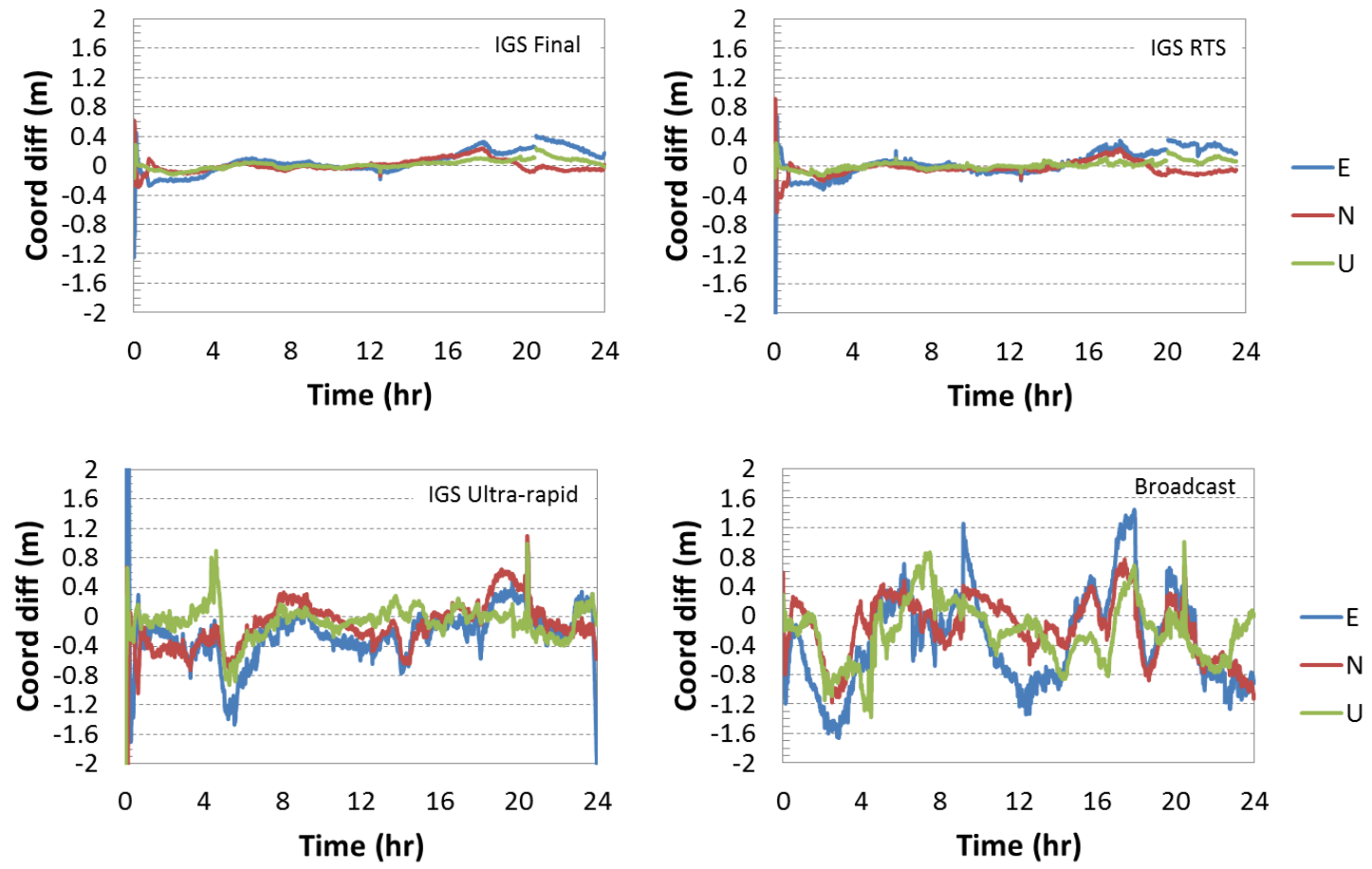

Figure 1 position accuracy using IGS final (top-left), IGS RTS (top-right), IGU (bottom-left) and Broadcast ephemerids (bottom-right) at station TWFT on 2/1/2017

Figure 1 and Table 1 show that the PPP using IGS RTS products gave results very close to those when using the final products. The drop in accuracy is clear if the user loses the RTS products and switches to the stored ultra-rapid product, where the mean error and the standard deviations are mostly more than doubled. Naturally, these were much worsening if the broadcast ephemeris and clock corrections were used.

For the implementation of the openly available IGS-RTS products, they are typically streamed in the Radio Technical Commission for Maritime Services (RTCM) - State Space Representation (SSR) format. The open source client application BNC (the German Federal Agency for Cartography and Geodesy -BKG- Network Transport of the Internet Protocol client, NTRIP) and the RTKLIB software package allow access to these corrections. The orbit corrections in RTCM-SSR are defined in terms of the radial, along-track and cross-track components, denoted as $\delta \rho_{r}, \delta \rho_{a}$ and $\delta \rho_{c}$ respectively, in addition to their velocities El-Mowafy, A. (2017). Impact of Predicting Real-Time Clock Corrections during their Outages on Precise Point Positioning. Survey Review, DOI 10.1080/00396265.2017.1405155. 
$\left(\delta \dot{\rho}_{r}, \delta \dot{\rho}_{a}\right.$ and $\left.\delta \dot{\rho}_{c}\right)$. Using a broadcast navigation message with a reference time $t_{o}$, the orbit corrections at time $t$ can be computed as follows (Hadas and Boys 2015):

$$
\delta \rho=\left[\begin{array}{lll}
\rho_{r} & \delta \rho_{a} & \delta \rho_{c}
\end{array}\right]^{T}+\left[\begin{array}{lll}
\delta \dot{\rho}_{r} & \delta \dot{\rho}_{a} & \delta \dot{\rho}_{c}
\end{array}\right]^{T}\left(t-t_{o}\right)
$$

These corrections are transformed to geocentric corrections by applying the radial, alongtrack and cross-track unit vectors $\left(e_{r}, e_{a}\right.$ and $\left.e_{c}\right)$ and adding them to the broadcast orbit $x_{\text {brdcst }}$ gives the final precise orbit $x_{\text {precise }}$ :

$$
x_{\text {precise }}=x_{\text {brdcst }}+\operatorname{diag}\left(e_{r} e_{a} e_{c}\right) \delta \rho
$$

The clock correction for each satellite is given as a range correction in terms of a quadratic polynomial with coefficients $(q 0, q 1, q 2)$ such that:

$$
c \delta t=q 0+q 1\left(t-t_{o}\right)+q 2\left(t-t_{o}\right)^{2}
$$

where $c$ is the speed of light. Then, the corrected satellite clock offset $t_{s a t}$ is expressed as:

$$
t_{s a t}=t_{b r d c s t}+\delta t
$$

where $t_{\text {brdcst }}$ denotes the broadcast GNSS satellite clock offset.

\section{Prediction models}

In this section, the proposed method used in place of the IGS-RTS orbit and clock corrections when they experience a break in their acquisition is discussed.

\section{Dealing with breaks in the orbit corrections}

In recent research, the GNSS orbits were predicted by using a polynomial fitting such as shown in Seppänen et al. (2012) and Hadas \& Bosy (2015). The latter achieved less than 10 $\mathrm{cm}$ accuracy for a prediction of RTS orbits for a period of up to 10 minutes, which is a relatively short period. In El-Mowafy et al. (2017) the orbital corrections were predicted instead of predicting the orbits themselves, i.e. predicting the difference between the broadcast and precise orbits. The multiplicative Holt-Winters' method was used for prediction of this difference, which comprises a level component, a trend component at each period and a third component representing seasonal changes (Chatfield and Yar 1991; Koehler et al. 1999; El-Mowafy, 2006). When an outage in RTS orbits occur, the forecast values are added to the broadcast orbits. A prediction error in the range $10-50 \mathrm{~cm}$ can result from this approach for the first $0.5 \mathrm{hr}$ of prediction and afterwards the prediction error significantly grows with time.

A simpler and practical solution for long orbital prediction periods is to download the most recent IGS ultra-rapid products (IGU), and use its orbits in case of experiencing a break in receiving the orbit corrections. The IGU is released four times each day and contains two days of orbits; the first day is computed from observations and the second day includes predicted orbits and clocks that can be used for RT applications (Dow et al., 2009). The differences between the IGU (predicted half) and the IGS final orbits, considered as a El-Mowafy, A. (2017). Impact of Predicting Real-Time Clock Corrections during their Outages on Precise Point Positioning. Survey Review, DOI 10.1080/00396265.2017.1405155. 
benchmark for precise orbits, are within $\pm 7 \mathrm{~cm}$ with standard deviations of $5 \mathrm{~cm}$ (El-Mowafy et al., 2017). Replacing the IGS RTS orbits by the predicted part of the IGU orbits is acceptable keeping in mind that the IGS RTS orbits are produced by polynomial fitting of the IGU orbits. Accordingly, the IGU and IGS RTS proved to be numerically and statistically compatible as explained in our earlier work in El-Mowafy et al. (2017). For example, the differences between IGU and IGS RTS orbits for all satellites in August 2015 were found to be mostly within $\pm 6 \mathrm{~cm}$. Statistical testing were performed by studying the significance of the residuals between the IGS final orbits and the two data streams (IGU and IGS RTS) using nonparametric statistical hypothesis tests. The Wilcoxon signed-rank test for the (IGS final orbits - IGU orbits) and (IGS Final orbits - IGS RTS orbits) daily residuals over August 2015 was tested to evaluate whether their population mean ranks differ. The test was successful for 99\% of the samples. In addition, testing of their variances was performed using KruskalWallis $\mathrm{H}$ test (Kruskal and Wallis 1952), which was successful for 93\% of the data with P values $>0.05$.

On the other hand, the IGU's clock corrections have RMS of almost 3 ns (Dow et al., 2009), which is not good enough for precise positioning. Therefore, the IGU clock corrections are not used when experiencing an outage in the RTS products, and instead the IGS RTS clock corrections are predicted as a time series as will be discussed in the next section.

\section{Prediction model of the clock corrections}

For prediction of clock corrections, we use a model comprising a linear component and sinusoidal terms expressed as:

$$
\delta t_{\text {predicted }}=a_{0}+a_{1} \Delta T+\sum_{i=0}^{4} A_{i} \sin \left(\frac{\Delta t_{i}}{\lambda_{i}} \times 2 \pi+\frac{t_{\phi_{i}}}{\lambda_{i}} \times 2 \pi\right)+\varepsilon_{\delta t}
$$

where $\delta t_{\text {predicted }}$ is the predicted clock correction, $\Delta T$ is the time since start of prediction, $a_{0}$ and $a_{1}$ are the bias and drift of the clock corrections, respectively, and $\varepsilon_{\delta t}$ denotes the noise. We consider four sinusoidal periods that proved to be most significant based on spectral analysis of the IGS-RTS clock corrections, using for instance Fast Fourier Transform, as shown in El-Mowafy et al. (2017). These periods are $15 \mathrm{~min}, 30 \mathrm{~min}, 3 \mathrm{hrs}$ and $12 \mathrm{hrs}$. The latter period is attributed to estimation of the clock corrections along with estimation of the orbital corrections. $A_{i}$ is the amplitude of period $i, \Delta t_{i}$ is the time since start of this period, and $t_{\phi_{i}}$ is its initial phase in time units. $\lambda_{i}$ is the time length for the periodic term $i$ (i.e. 15 min, $30 \mathrm{~min}, 3 \mathrm{hrs}$, and $12 \mathrm{hrs}$ for $i=1$ to 4 ). In practice, $\delta t_{\text {predicted }}$ is used whenever an outage of real-time products is experienced. The parameters of the prediction model (i.e. $a_{0}$, $a_{1}, A_{i}$ and $t_{\phi_{i}}$ ) are determined using least squares for a period of 1-2 hrs of data. To reduce the computation load, the model is built with a sliding time window, preferably applied at a certain interval, such as 15 minutes.

Prediction of clock corrections as a time series requires the data used in building the model to be outlier free. Figure 2 shows the 30 seconds epoch-differenced clock corrections (i.e. the difference between clock corrections of two consecutive epochs) for GPS PRNs 16, 29 and 30 on two days, one week apart, where the top panel shows the data of 17/6/2016 and the lower panel illustrates the data of 24/6/2016. As can be seen, there are jumps in the epochdifferenced clock corrections, which are almost the same for the clocks of all satellites in the same day, but they differ between days. Therefore, these jumps in the clock corrections can El-Mowafy, A. (2017). Impact of Predicting Real-Time Clock Corrections during their Outages on Precise Point Positioning. Survey Review, DOI 10.1080/00396265.2017.1405155. 
be attributed to a common source for all satellites, i.e. an Analysis Centre (AC) Reference time offset (a bias). Similar clock jumps were observed by Mervart and Weber (2011) and Chen et al. (2017). This offset is due to that the clock products are generated at the contributing ACs (currently eight ACs) using their inherent time scales, and the final RTS product is referenced to one of the ACs. This reference AC may change at any time according to availability and quality of the generated products, and accordingly a jump in RTS clock corrections may take place when switching from one reference AC to another AC (personal communications with IGS RTS working group). For PPP processing, the common part of this offset, for instance the mean value for all satellites at each epoch, can be absorbed in the estimated receiver clock offset that is being determined each epoch. Thus, PPP results remain unaffected by this offset. The remaining clock differences from the common offset value, which vary among different satellites typically within $\pm 2 \mathrm{~ns}$, can be absorbed in the individual satellite ambiguities. However, since the time series of the clock corrections for each individual satellite are used in building the prediction model, these jumps in the clock corrections have to be detected and accounted for.
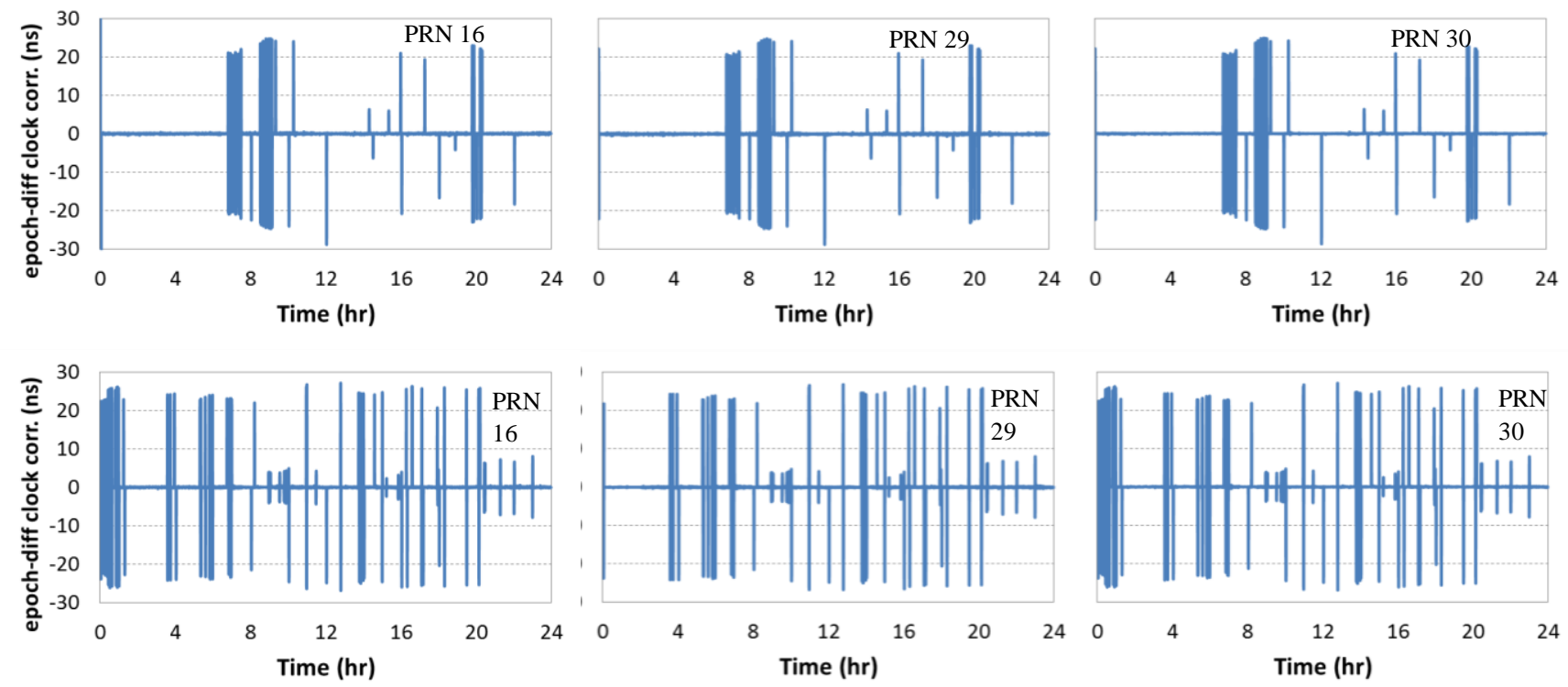

Figure 2 Epoch-differenced clock corrections for GPS PRN 16 (left), PRN 29 (middle) and PRN 30 (right). Top row is for 17/6/2016 and bottom row is for 24/7/2016

An outlier is suspected at epoch $t$ when the following condition is met:

$$
|\Delta \delta t(t)-M(t-1)|>K \sigma_{\Delta \delta t}
$$

where $|\Delta \delta t(t)-M(t-1)|$ is the test statistic, $\Delta \delta t(t)$ is the epoch-differenced IGS-RTS clock correction at $t$, and $M(t-1)$ is the moving average of $\Delta \delta t$ computed at epoch $(t-1)$. $M(t-1)$ is computed for a selected sliding window of $n$ epochs, e.g. $n=30$, as follows:

$$
M(t-1)=M(t-2)+\frac{1}{n}(\Delta \delta t(t-1)-M(t-2))
$$

El-Mowafy, A. (2017). Impact of Predicting Real-Time Clock Corrections during their Outages on Precise Point Positioning. Survey Review, DOI 10.1080/00396265.2017.1405155. 
$K \sigma_{\Delta \delta t}$ is the test threshold, where $\sigma_{\Delta \delta t}$ is the standard deviation of the statistic computed over a long period of time. Figure 3 shows example histograms of $\Delta \delta t$ for PRNs 16, 29 and 30 for one day of data (17/07/2017), which shows that their distribution can approximately resemble a normal distribution. Accordingly, $K$ is taken as the inverse of the complement of the one-sided standard normal cumulative distribution function for a selected confidence interval. For instance, for a confidence interval $99.7 \%, K$ is 3 . An alternative approach for detection of outliers is to use the single-channel validation method presented in El-Mowafy, 2014 and 2015. Once an outlier data is detected, it is excluded from the computation in the prediction model, noting here that the model presented in Eq. (5) does not require the use of continuous data. The jumps in RTS clock corrections when switching among ACs can be identified when the check in (6) fails for an extended period of time due to the depart of clock corrections from their moving average, whereas the difference in the clock corrections for consecutive epochs lies within three times their standard deviation (taking $K=3$ as above). The moving average is adjusted accordingly for this bias. As stated earlier, this bias, which is common for all satellites from the same system, will be absorbed in the estimated receiver clock offset and thus will not affect positioning.
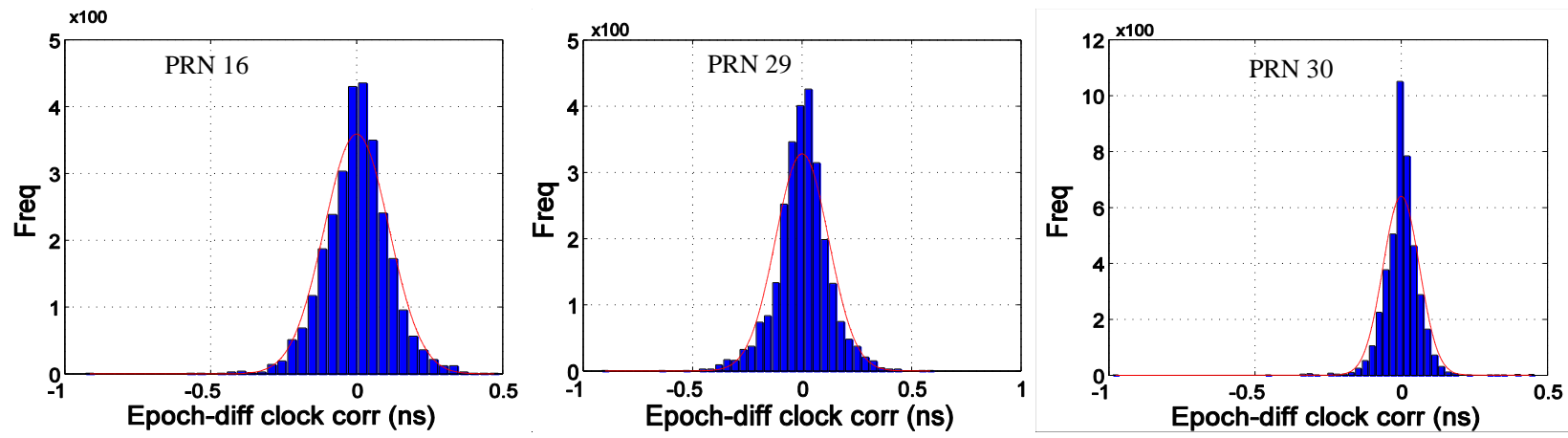

Figure 3 Histograms of epoch-differenced $\Delta \delta t$ for GPS PRN 16 (left), PRN 29 (middle) and PRN 30 (right) on 17/07/2016

In the prediction model, the outlier-free observations are weighted according to age of data, where the most recent observations are given more weights. The weight is assumed decaying gradually with time in the form:

$$
W=\operatorname{diag}\left(w_{j}\right), \quad w_{j}=e^{-\Delta t / T} \times 1 / \sigma_{\delta t_{j}}^{2}
$$

where $W$ is the weight matrix, $w_{j}$ is the individual weight for $\delta t$ number $j$, taken during the regression period used for the development of the model. $T$ is the correlation time length, which is determined from the autocorrelation analysis of the clock corrections. $\sigma_{\delta t}$ is set according to analysis of Allan deviations of the clock corrections as explained in our earlier work in El-Mowafy et al. (2017).

\section{Accuracy of clock prediction}

In this section, the accuracy of predicting IGS-RTS clock corrections using the presented model is evaluated. The IGC01 stream of the IGS-RTS was used, which is based on a single epoch GPS combination solution. Results of two days of data on 23 and 24 August 2015 are

El-Mowafy, A. (2017). Impact of Predicting Real-Time Clock Corrections during their Outages on Precise Point Positioning. Survey Review, DOI 10.1080/00396265.2017.1405155. 
used to demonstrate the performance of the proposed model. One hour of data was used in building the prediction model, i.e. estimation of the model coefficients. The prediction error is defined in terms of the difference between the predicted values of the clock corrections and their original values during the prediction period. Table 2 summarizes the statics of this prediction error of clock corrections for all satellites in the current three GPS blocks (II-R, IIRM, and II-F) during the first hour of prediction. The table shows that the STD of different blocks range between $0.13 \mathrm{~ns}$ and $0.25 \mathrm{~ns}$. To show the behaviour of the prediction error over a longer period, Figure 4 shows the prediction error for up to $3 \mathrm{hrs}$ for each of the test days. The figure clearly shows that the error increases with the increase of prediction time for all satellites. The prediction error was typically within $0.5 \mathrm{~ns}$ during the first hour and $1 \mathrm{~ns}$ after $2 \mathrm{hrs}$. In addition, the satellites that have clock corrections causing most of the errors belong to the old generation of blocks II-R or II-RM. Furthermore, prediction of PRN 24, which is the only satellite that has a caesium clock was much worse than the rest of the satellites which have rubidium clocks. Therefore, PRN 24 was excluded from the computation of the statistics presented in Table 2 and in positioning when predicting the clock corrections.

Since the prediction error grows with time, one has to consider age of the model, defined as the time since the last epoch when the prediction model parameters were estimated. For instance, if one is seeking errors less than a specific value (e.g. $1 \mathrm{~ns})$, the estimated model parameters should not be older than a certain time, e.g. $1 \mathrm{hr}$, and should only be valid for a certain period, e.g. $1 \mathrm{hr}$. This period is inversely proportional with the age of the model. This age and validity period should be defined according to the user needs and the application at hand.
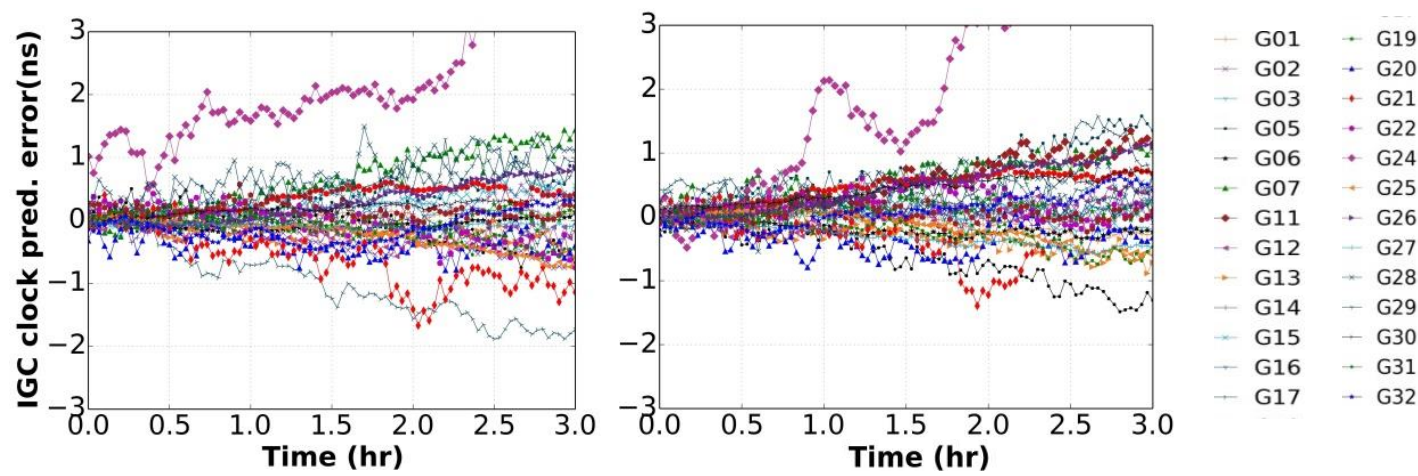

Figure 4 Prediction error of IGS-RTS clock corrections for August 23 and 24, 2015

Table 2 Statics of the prediction error (in ns) of the IGS-RTS clock corrections for all GPS blocks for the first hour of prediction

\begin{tabular}{ccccc}
\hline & \multicolumn{4}{c}{ Block } \\
\hline Block & II-R & II-RM & II-F & All \\
\hline mean & 0.02 & 0.10 & 0.03 & 0.04 \\
\hline STD & 0.28 & 0.21 & 0.13 & 0.25 \\
\hline Max (absolute) & 1.05 & 0.68 & 0.47 & 1.05 \\
\hline
\end{tabular}

El-Mowafy, A. (2017). Impact of Predicting Real-Time Clock Corrections during their Outages on Precise Point Positioning. Survey Review, DOI 10.1080/00396265.2017.1405155. 


\section{The impact of prediction of the clock corrections on PPP results}

The previous sections show that the use of IGU predicted orbits and predicting IGC clock corrections would represent a useful substitute to IGS-RTS corrections when the connection with the stream caster is lost for a short to medium period, e.g. $1 \mathrm{hr}$. In this case, a real-time user can use the precise IGS ultra-rapid (IGU) orbits as $x_{\text {precise }}$ in Eq. (2) and implement $\delta t_{\text {predicted }}$ in Eq. (4). This will require the user to download the most recent IGU orbits. For testing practical application of the proposed method, its performance was evaluated at five IGS stations that have a global distribution; SASK (Canada), HERT (United Kingdom), PTBB (Germany), GMSD (Japan), and PERT (Australia). The data used spanned one week (2-8 January, 2017) with a sampling interval of $30 \mathrm{~s}$, and comprised ionosphere-free combination of L1 and L2 dual-frequency GPS observations. The data was processed in a float-ambiguity PPP approach by using Kalman filter in a kinematic mode, i.e. determine the positions at each epoch. The models used in data processing are given in Table 3 . The IGC01 clock corrections were predicted for one hour, assumed as a period of an outage in receiving the IGS-RTS products. The prediction model parameters were estimated through a regression period of $1 \mathrm{hr}$ before the assumed break. The tests were applied for each of the tested sites 6 times per each day, at 4 hours interval, for the seven days of testing. The impact of using the proposed prediction model is assessed for two aspects; solution convergence time and positioning accuracy. Their results are discussed in the next sections.

Table 3. Kalman Filter design of the used PPP model

\begin{tabular}{|c|c|c|c|c|}
\hline State & Initial value & Initial STD & $\begin{array}{l}\text { Dynamic } \\
\text { model }\end{array}$ & $\begin{array}{l}\text { Process noise } \\
\text { STD }\end{array}$ \\
\hline $\begin{array}{l}\text { Position } \\
\text { coordinates } \\
(\mathrm{E}, \mathrm{N}, \mathrm{U})\end{array}$ & $\begin{array}{l}\text { From least- } \\
\text { squares single } \\
\text { point positioning }\end{array}$ & $5 \mathrm{~m}$ & Random walk & $\begin{array}{c}1 \mathrm{~m} / \sqrt{\mathrm{s}} \\
\text { (kinematic) }\end{array}$ \\
\hline $\begin{array}{l}\text { Receiver clock } \\
\text { offset }\end{array}$ & $\begin{array}{l}\text { From least- } \\
\text { squares single } \\
\text { point positioning }\end{array}$ & $\begin{array}{l}1 \mathrm{~ms} \text { (milli- } \\
\text { second) }\end{array}$ & Random walk & $10^{6} \mathrm{~m} / \sqrt{\mathrm{s}}$ \\
\hline Troposphere & $\begin{array}{l}\text { From empirical } \\
\text { models, .e.g. } \\
\text { Hopfield }\end{array}$ & $0.1 \mathrm{~m}$ & $\begin{array}{l}\text { 1st order Gauss- } \\
\text { Markov, Time } \\
\text { constant }=2 \mathrm{hrs}\end{array}$ & $10^{-6} \mathrm{~m} / \sqrt{\mathrm{s}}$ \\
\hline $\begin{array}{l}\text { Phase } \\
\text { ambiguities }\end{array}$ & $\begin{array}{l}\text { from estimated } \\
\text { code range }\end{array}$ & 100 cycle & constant & 0 \\
\hline
\end{tabular}

\section{The impact of prediction on PPP solution convergence}

Since the float-ambiguity PPP is a positioning method that is characterised by a long initialisation period for convergence of the solution and ambiguities (Deo and El-Mowafy, 2017), two cases were considered to evaluate the impact of using the predicted clocks and IGU orbits. The first case represents a typical scenario, where a break in communications and in receiving the real-time corrections is assumed taking place during normal PPP operation, e.g. after $1 \mathrm{hr}$ from start, where the PPP solution has already converged. In this study, the convergence time is defined as the first time that the STDs for all coordinate components reached $10 \mathrm{~cm}$ or less and maintained this level. The second case resembles a critical scenario, where it is assumed that a disturbance in the hardware took place, resulting in El-Mowafy, A. (2017). Impact of Predicting Real-Time Clock Corrections during their Outages on Precise Point Positioning. Survey Review, DOI 10.1080/00396265.2017.1405155. 
resetting of the GNSS receiver and a restart of the initialisation while the communication with real-time corrections was not regained. Thus, the immediate stored values of the prediction model are used, which lay within the acceptable model validity period, to predict the clock corrections until communication with IGS-RTS products is recovered. Hence, the predicted clock corrections will affect the initialisation process and convergence of the ambiguities as well as the obtained positions.

For illustration, the STDs $\left(\sigma_{\mathrm{E}}, \sigma_{\mathrm{N}}\right.$ and $\left.\sigma_{\mathrm{U}}\right)$ of the local-level E, $\mathrm{N}$ and $\mathrm{U}$ coordinates for the two cases are depicted in Figure 5 for one test at station GMSD. The average of the STDs and convergence times, for all stations and for all tests, are given in Table 4. In addition, the average results of PPP without prediction using the original IGS RTS products are given in the last row of the table as a reference to show the expected performance if no break in the corrections was experienced. Results show that the proposed method resulted in PPP at a precision of all coordinates less than $10 \mathrm{~cm}$ after solution convergence, which were slightly less than the precision of PPP results without prediction (shown in the last row of Table 4). When PPP was re-initialized at the break and using only the predicted clock corrections, the initialization period has increased by almost 10-15\% and the STDs were adversely affected by the use of the predicted products. However, the STDs were still less than a decimetre after convergence. The positioning errors, computed as the difference between the estimated PPP position and the known station position, after solution convergence for the two cases are listed in Table 5. Noting that the prediction error grows with time, the slight increase in positioning errors for the case when the break took place at start of initialization is attributed to using delayed portion of the predicted clocks, whereas for the same test period in the first case (where a break is assumed after convergence) an early portion of the predicted values was used.
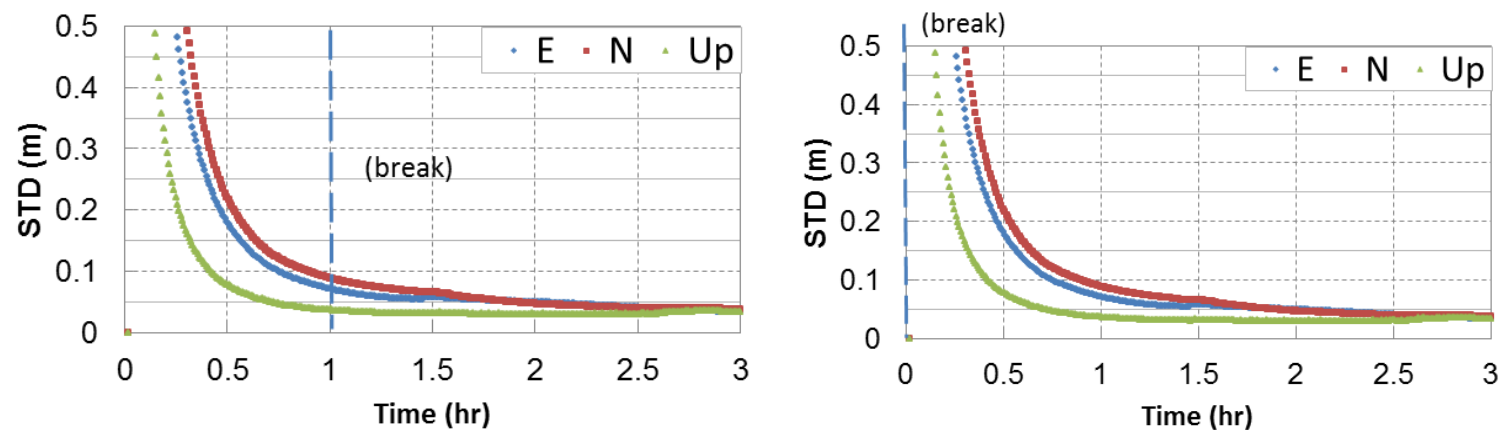

Figure 5 PPP STD in Easting (E), Northing (N) and Up (U) using IGU orbits and predicted IGC01 clock corrections for one example at station GMSD. PPP starts at $1 \mathrm{hr}$ after the break (left) and at the break (right) 
Table 4 Average statistics of PPP using IGU orbits and predicted IGC clock corrections with breaks after convergence and at initialisation

\begin{tabular}{|c|c|c|c|c|}
\hline \multirow{2}{*}{ Break occurrence } & \multirow{2}{*}{$\begin{array}{l}\text { Convergence } \\
\text { period (min) }\end{array}$} & \multicolumn{3}{|c|}{$\begin{array}{l}\text { average STDs after } \\
\text { convergence }\end{array}$} \\
\hline & & $\sigma_{\mathrm{E}}$ & $\sigma_{\mathrm{N}}$ & $\sigma_{\mathrm{U}}$ \\
\hline After convergence & 42 & 0.049 & 0.052 & 0.063 \\
\hline At start of initialization & 46 & 0.052 & 0.055 & 0.066 \\
\hline PPP without a break & 39 & 0.040 & 0.044 & 0.066 \\
\hline
\end{tabular}

Table 5 Average values of the position error using IGS RTS with and without prediction

\begin{tabular}{c|cccccc}
\hline Case & \multicolumn{3}{|c}{ Break after convergence } & \multicolumn{4}{c}{$\begin{array}{c}\text { Break at start of } \\
\text { initialization }\end{array}$} \\
\hline component & $\mathrm{E}$ & $\mathrm{N}$ & $\mathrm{U}$ & $\mathrm{E}$ & $\mathrm{N}$ & $\mathrm{U}$ \\
\hline $\begin{array}{c}\text { Mean } \\
\text { (absolute) }\end{array}$ & 0.083 & 0.092 & 0.094 & 0.115 & 0.125 & 0.126 \\
\hline
\end{tabular}

\section{The impact of prediction on PPP positioning accuracy and precision}

To show the impact of the use of the predicted orbit and clock corrections on PPP positioning accuracy and precision we compared its performance when prediction was applied versus the case when using the original corrections without prediction. Furthermore, as discussed earlier, during an outage of the IGS RTS products, the best available option for a user at present is to exploit the IGU products. While the same orbit information are used in the proposed method as with the IGU, prediction of the clock corrections of the IGS RTS instead of using the IGU clock corrections will make a substantial difference for the final positions. Therefore, the potential improvement in positioning due to the use of the proposed method was additionally evaluated versus the use of IGU clocks. Accordingly, during an assumed break for the tested station data, and using the same sets of observations for the three cases, PPP positions were compared, namely using: IGS RTS without prediction, IGS RTS predicted clocks + IGU orbits, and IGU products. The accuracy of the computed PPP coordinates in each case was evaluated in terms of the position error, defined as the difference between the estimated PPP coordinates and the known station coordinates.

Figure 6 shows the time series of the position errors in E, $N$ and $U$ coordinates in the locallevel frame for one test period as a representative example for the three compared cases at the test stations, showing results of HERT ( $1^{\text {st }}$ panel); GMSD $\left(2^{\text {nd }}\right.$ panel $)$; PERT ( $3^{\text {rd }}$ panel); PTBB ( $4^{\text {th }}$ panel); and SASK $\left(5^{\text {th }}\right.$ panel). Since the prediction error grows with time, we restrict attention here on results when applying the prediction model for $1 \mathrm{hr}$, assuming a communication break can be restored within this time period. The shown example is for the period 2:00-3:00 GPS time on $2^{\text {nd }}$ January, 2017. The mean results for all tested periods (6 test periods each day for 7 days) at each of the test sites are given in the Tables 6 to 8. Table 6 shows the mean of the absolute values of the PPP errors computed at each epoch and Table 7 gives their RMSE after convergence. The two tables demonstrate the obtainable accuracy. Table 8 depicts the solution precision after convergence in terms of the computed STDs. As

El-Mowafy, A. (2017). Impact of Predicting Real-Time Clock Corrections during their Outages on Precise Point Positioning. Survey Review, DOI 10.1080/00396265.2017.1405155. 
illustrated, the results of using IGS-RTS without prediction were better than with prediction, and both were significantly better than when using IGU. The mean absolute position errors using the original corrections were less than $10 \mathrm{~cm}$ and when using the predicted corrections, the mean values of absolute errors were mostly less than $20 \mathrm{~cm}$, with RMSE generally better than $15 \mathrm{~cm}$ and a precision within a decimetre. Additionally, the improvement gained from using the proposed method compared with the use of IGS ultra-rapid products was in general significant except for a few cases where they gave close results. For instance, the shown case at station GMSD, which has good satellite geometry. Overall, the reduction in the mean position errors and the RMSE when using the predicted IGS-RTS clocks versus when using IGU clocks ranged approximately from $20 \%$ to $60 \%$ as depicted in the Tables 6 and 7.

Table 6 Mean of the absolute positioning errors (in $\mathrm{m}$ ) of all tested sessions after solution convergence

\begin{tabular}{|c|c|c|c|c|c|c|c|c|c|}
\hline \multirow[t]{2}{*}{ Station } & \multicolumn{3}{|c|}{ IGS RTS } & \multicolumn{3}{|c|}{$\begin{array}{l}\text { IGS RTS predicted } \\
\text { clocks + IGU orbits }\end{array}$} & \multicolumn{3}{|c|}{ IGU orbits + clocks } \\
\hline & $\mathrm{E}$ & $\mathrm{N}$ & $\mathrm{U}$ & $\mathrm{E}$ & $\mathrm{N}$ & $\mathrm{U}$ & $\mathrm{E}$ & $\mathrm{N}$ & $\mathrm{U}$ \\
\hline HERT & 0.054 & 0.061 & 0.075 & 0.103 & 0.076 & 0.161 & 0.216 & 0.192 & 0.377 \\
\hline GMSD & 0.071 & 0.055 & 0.078 & 0.083 & 0.092 & 0.094 & 0.269 & 0.165 & 0.336 \\
\hline PERT & 0.057 & 0.078 & 0.097 & 0.138 & 0.188 & 0.206 & 0.307 & 0.292 & 0.476 \\
\hline PTBB & 0.059 & 0.043 & 0.069 & 0.139 & 0.103 & 0.206 & 0.216 & 0.257 & 0.313 \\
\hline SASK & 0.038 & 0.064 & 0.085 & 0.074 & 0.083 & 0.152 & 0.127 & 0.146 & 0.242 \\
\hline
\end{tabular}

Table 7 Mean of the positioning RMSE (m) for all tested sessions after solution convergence

\begin{tabular}{|c|c|c|c|c|c|c|c|c|c|}
\hline \multirow[t]{2}{*}{ Station } & \multicolumn{3}{|c|}{ IGS RTS } & \multicolumn{3}{|c|}{$\begin{array}{l}\text { IGS RTS predicted } \\
\text { clocks + IGU orbits }\end{array}$} & \multicolumn{3}{|c|}{ IGU orbits + clocks } \\
\hline & $\mathrm{E}$ & $\mathrm{N}$ & $\mathrm{U}$ & $\mathrm{E}$ & $\mathrm{N}$ & $\mathrm{U}$ & $\mathrm{E}$ & $\mathrm{N}$ & $\mathrm{U}$ \\
\hline HERT & 0.052 & 0.061 & 0.053 & 0.137 & 0.068 & 0.154 & 0.257 & 0.237 & 0.264 \\
\hline GMSD & 0.063 & 0.060 & 0.077 & 0.064 & 0.086 & 0.086 & 0.148 & 0.189 & 0.249 \\
\hline PERT & 0.051 & 0.112 & 0.093 & 0.119 & 0.166 & 0.177 & 0.269 & 0.259 & 0.342 \\
\hline PTBB & 0.056 & 0.049 & 0.062 & 0.139 & 0.079 & 0.183 & 0.212 & 0.240 & 0.321 \\
\hline SASK & 0.035 & 0.046 & 0.083 & 0.077 & 0.105 & 0.117 & 0.178 & 0.147 & 0.180 \\
\hline
\end{tabular}

Table 8 Mean of the position STD (m) for all tested sessions after solution convergence

\begin{tabular}{|c|c|c|c|c|c|c|c|c|c|}
\hline \multirow[t]{2}{*}{ Station } & \multicolumn{3}{|c|}{ IGS RTS } & \multicolumn{3}{|c|}{$\begin{array}{l}\text { IGS RTS predicted } \\
\text { clocks + IGU orbits }\end{array}$} & \multicolumn{3}{|c|}{ IGU orbits + clocks } \\
\hline & $\sigma_{\mathrm{E}}$ & $\sigma_{N}$ & $\sigma_{U}$ & $\sigma_{\mathrm{E}}$ & $\sigma_{N}$ & $\sigma_{U}$ & $\sigma_{\mathrm{E}}$ & $\sigma_{N}$ & $\sigma_{U}$ \\
\hline HERT & 0.054 & 0.046 & 0.058 & 0.054 & 0.046 & 0.057 & 0.060 & 0.049 & 0.072 \\
\hline GMSD & 0.058 & 0.063 & 0.063 & 0.057 & 0.063 & 0.079 & 0.057 & 0.063 & 0.066 \\
\hline PERT & 0.041 & 0.079 & 0.087 & 0.044 & 0.078 & 0.084 & 0.064 & 0.069 & 0.081 \\
\hline PTBB & 0.048 & 0.044 & 0.056 & 0.047 & 0.044 & 0.052 & 0.058 & 0.045 & 0.065 \\
\hline SASK & 0.039 & 0.043 & 0.056 & 0.039 & 0.042 & 0.046 & 0.049 & 0.046 & 0.049 \\
\hline
\end{tabular}

El-Mowafy, A. (2017). Impact of Predicting Real-Time Clock Corrections during their Outages on Precise Point Positioning. Survey Review, DOI 10.1080/00396265.2017.1405155. 

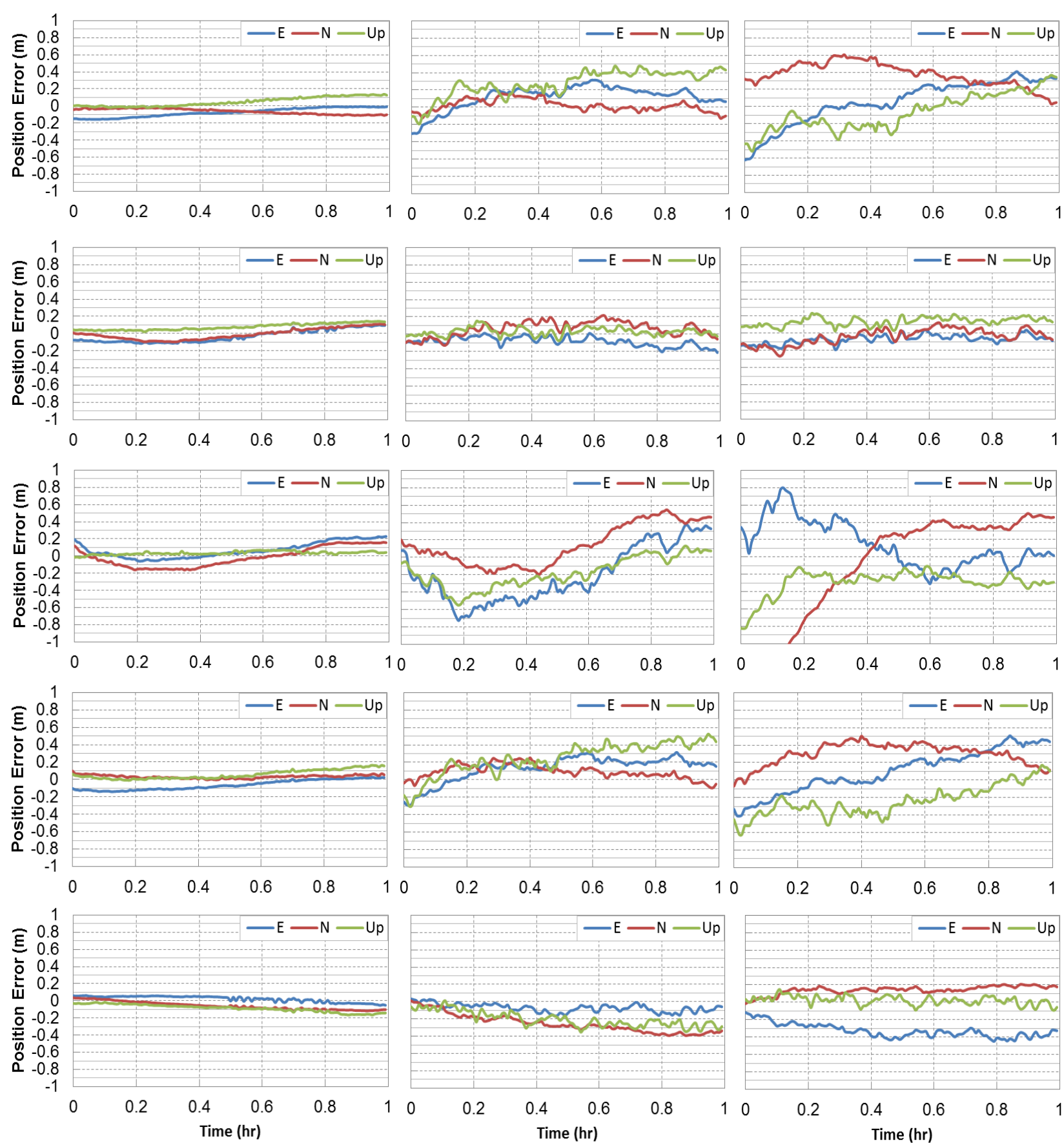

Figure 6 PPP errors for one example using IGS RTS without prediction (left), IGS RTS predicted clocks + IGU orbits (middle) and IGU products (right) - during the period 2:003:00 GPS time on $02 / 01 / 2017$, for stations HERT ( ${ }^{\text {st }}$ panel $)$; GMSD $\left(2^{\text {nd }}\right.$ panel $)$; PERT $\left(3^{\text {rd }}\right.$ panel $)$; PTBB ( $4^{\text {th }}$ panel $)$; and SASK $\left(5^{\text {th }}\right.$ panel $)$.

El-Mowafy, A. (2017). Impact of Predicting Real-Time Clock Corrections during their Outages on Precise Point Positioning. Survey Review, DOI 10.1080/00396265.2017.1405155. 


\section{Conclusion}

It is shown that an outage in receiving the real-time orbit and clock corrections can result in a significant degradation in PPP positioning. A method is proposed to bridge this outage and maintain positioning accuracy at less than 2 decimetres. In this method, the IGS ultra-rapid orbits are used, which are numerically and statistical compatibility with the real-time orbits, and the GPS clock corrections are predicted as a time series. The clock prediction model comprises a linear component and four major sinusoidal terms. The error due to clock prediction grows with the increase of prediction time. It was typically within $0.5 \mathrm{~ns}$ during the first hour and $1 \mathrm{~ns}$ after $2 \mathrm{hrs}$. Due to this behaviour, the user has to consider age of the model, defined here as the time since the last epoch when the prediction model was built, and the period of model validity, which is inversely proportional to age of the model. This age and validity periods should be defined according to the user needs and application.

The use of predicted clocks increased the convergence period of PPP by about 10-15\%, when considering a critical case where PPP was initialized at the break. In order to validate the proposed approach, a comparison was made between the PPP errors, computed as the difference between the estimated PPP position and the known station coordinates, at three cases, namely: when using IGS RTS without prediction, when employing IGS RTS predicted clocks + IGU orbits, and when using IGU products. The same sets of observations were used in processing the same data set when implementing the three products. Results at five IGS stations of a global distribution showed that position errors using the original corrections were typically less than $10 \mathrm{~cm}$, and when using the predicted corrections the mean of the absolute errors was mostly less than $20 \mathrm{~cm}$ with RMSE generally less than $15 \mathrm{~cm}$ and STDs less than a decimetre. Currently, the best traditional method in case of experiencing a break in receiving the real-time corrections is to use the IGS ultra-rapid products. The improvement gained from using the proposed method compared with this traditional approach is quite evident, where the mean position errors and the RMSE were reduced approximately between $20 \%$ and $60 \%$.

\section{Acknowledgment}

The data used for this study was obtained from open source streams provided by IGS (Dow et al., 2009) and IGS RTS which we would like to gratefully acknowledge. The data providers of stations TWFT, GSMD, PERT, PHBB, HERT, and SASK are acknowledged. The author would like to thank Mr Manoj Deo for processing some of the data used. This study is supported by an Australian Research Council project number DP170103341.

\section{References}

Bawden GW, Melbourne TI, Bock, Y. et al., 2016. Development of a GNSS-Enhanced Tsunami Early Warning System. 11th Meeting of the International Committee on GNSS - Sochi, Russia, 07 - November, 2016, 1-36.

Blewitt G, Hommond W, Kreemer, C et al., 2009. GPS for real-time earthquake source determination and tsunami warning systems, J of Geodesy 83:335-343.

Chen L, Song W, Yi W, Shi C, Lou Y, Guo H, 2017. Research on a method of real-time combination of precise GPS clock corrections. GPS Solutions 21:187-195

Chatfield C, Yar M, 1991. Prediction Intervals for Multiplicative Holt-Winters. International Journal of Forecasting 7, 31-37.

El-Mowafy, A. (2017). Impact of Predicting Real-Time Clock Corrections during their Outages on Precise Point Positioning. Survey Review, DOI 10.1080/00396265.2017.1405155. 
Dow JM, Neilan, R E, Rizos C, 2009. The International GNSS Service in a changing landscape of Global Navigation Satellite Systems, Journal of Geodesy 83:191-198.

Deo M, El-Mowafy A, 2017. Triple-frequency GNSS models for PPP with float ambiguity estimation: performance comparison using GPS. Survey Review. doi.org/10.1080/ 00396265.2016.1263179

El-Mowafy A, 2006. On-the-fly Prediction of Orbit Corrections for RTK Positioning. J of Navigation 59(2): 321-334.

El-Mowafy A, 2014. GNSS Multi-Frequency Receiver Single-Satellite Measurement Validation Method. GPS Solutions 18(4):553-561.

El-Mowafy A, 2015. Estimation of Multi-Constellation GNSS Observation Stochastic Properties Using a Single-Receiver Single-Satellite Data Validation Method. Survey Review 47-341: 99-108.

El-Mowafy A, Deo M, Kubo N, 2017. Maintaining Real-Time Precise Point Positioning during Outages of Orbit and Clock Corrections, GPS Solutions 21(3), 937-947.

Hadas T, Bosy J, 2015. IGS RTS precise orbits and clocks verification and quality degradation over time. GPS Solutions 19(1): 93-105.

Hoechner A, Ge M. Babeyko A, Sobolev S V, 2013. Instant tsunami early warning based on real-time GPSTohoku 2011 case study. Nat. Hazards Earth Syst. Sci., 13, 1285-1292

Huang G W, Zhang Q, Xu GC, 2014. Real-time Clock offset prediction with an improved model. GPS Solutions, 18(1): 95-104.

Koehler A, Snyder R D, Ord J K, 1999. Forecasting Models and Prediction Intervals for the Multiplicative HoltWinters Method. Working Paper 1/99, Department of Economics and Business Statistics, Monash University, Australia.

Kruskal WH, Wallis A, 1952. Use of ranks in one-criterion variance analysis. J Am Stat Assoc 47(260):583-621.

Leandro R, Landau, H, Nitschke, M, et al., 2011. RTX Positioning: The Next Generation of cm-accurate RealTime GNSS Positioning. Proc. ION GNSS+ 2011, Inst. of Navigation, Portland, 20-23 Sept: 1460 - 1475

Mervart L, Weber G, 2011. Real-time combination of GNSS orbit and clock correction streams using a Kalman filter approach. In: Proc. ION GNSS+ 2011, Portland, OR, USA, 20-23 Sept 2011, 707-711.

Rizos C, 2011. Real-Time IGS in Support of Tsunami Early Warning. IGNSS Conference, Gold Coast, Australia, 14-16 July 2015, 1-30.

Saito E, Kubo N, 2016. Performance Evaluation and A New Disaster Prevention System of Precise Point Positioning at Sea, Proc. of ION GNSS+ 2016, Portland, Or, Sept. 12-16, 2016, 3412 - 3432.

Seppänen M, Ala-Luhtalaet J, Piché R. et al., 2012. Autonomous Prediction of GPS and GLONASS Satellite Orbits. Navigation 59(2): 119-134.

Yang H., Xu C, Gao Y. (2017): Analysis of GPS satellite clock prediction performance with different update intervals and application to real-time PPP, Survey Review, August 2017. DOI: 10.1080/00396265.2017.1359473.

Zumberge JF, Heflin MB, Jefferson DC, Watkins MM, Webb FH, 1997. Precise point positioning for the efficient and robust analysis of GPS data from large networks, J. Geophys. Res., 102(B3), 5005-5017.

El-Mowafy, A. (2017). Impact of Predicting Real-Time Clock Corrections during their Outages on Precise Point Positioning. Survey Review, DOI 10.1080/00396265.2017.1405155. 